\title{
Commissioned Review: The national strategy for sexual health and HIV: A perspective from Family Planning
}

Connie Smith, MFF, Services for Women, Parkside Health NHS Trust, St Charles Hospital, Exmoor Street, London W10 5SH

The Journal of Family Planning and Reproductive Health Care 2001: 27(4): 230-233

\section{Background}

Family planning services in England are approaching a watershed. The community Trusts from which the majority of family planning services are provided will largely cease to exist with the formation of the next wave of Primary Care Trusts (PCTs) in April 2002. After this the services will be both commissioned and provided from within these new organisations.

The creation of PCTs was designed to establish more integrated working between general practice and community health services, and offers opportunities to improve the quality of contraceptive care offered to local communities. However, some turbulence can be expected during the period of transition and the recent, much delayed, publication of the National Sexual Health/HIV Strategy consultation document provides a framework for local discussions on the future provision and development of contraceptive care in the National Health Service (NHS).

A summary of current contraceptive services provided by community and some hospital Trusts is offered by the annual Department of Health Statistical Bulletin. In 19992000 there were about 2.6 million attendances at family planning clinics ${ }^{1}$ (Figure 1). It is estimated that this represents about $20 \%$ of all contraceptive care, most of the remainder is provided from GP practices.

Figure 1 Clinic attendances 1999-2000. Rate (\%) per 100 resident women (Source: Statistical Bulletin) ${ }^{1}$

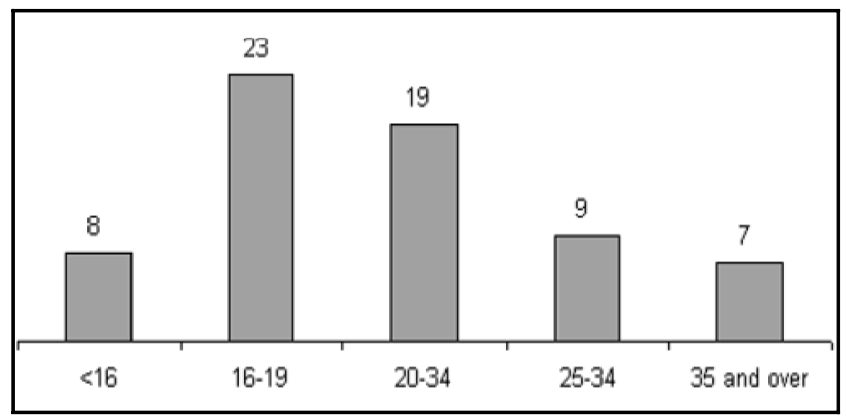

The distribution of family planning clinics has, to date, been determined largely by the history of local champions (including clinicians, service managers and public health physicians) and the interest (or otherwise) of local purchasers. This has resulted in variations, producing inequalities in the range, quality and accessibility of service provision across England. ${ }^{1}$ [4.3] The pattern of services does not seem to reflect the pattern of need.

The variation in the size of community family planning services is very marked, with annual attendances from $<1000$ to $>70000$ between services $^{1}$ (Figure 2).

There is no routine analysis of the contraceptive care provided by GP practices, so it is difficult to assess the extent to which family planning services are filling any gaps in local provision.
Figure 2 Clinic activity by region 1999-2000 (Source: Statistical Bulletin) ${ }^{1}$

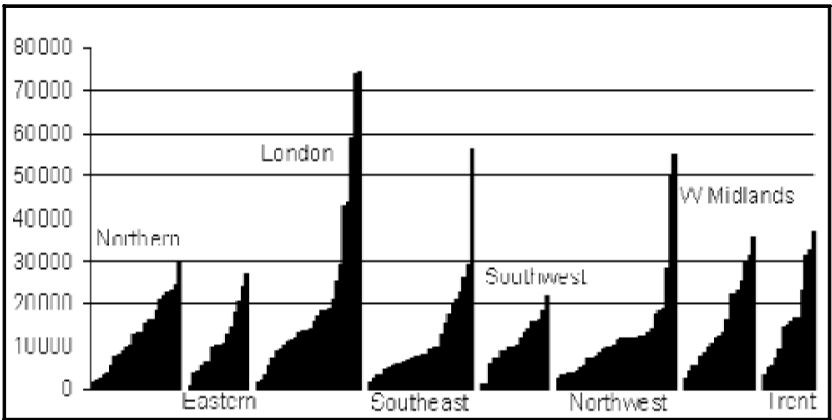

Despite the free availability of contraceptive care from most GP practices and the many family planning clinics, the rate of unwanted pregnancy, as reflected in the abortion rate (nearly 174000 in 1999), demonstrates that services are not meeting the challenges of providing contraceptive care for all who need it.

There are now over a million annual visits to departments of genito-urinary medicine (GUM), and an unknown number of consultations about sexually transmitted infections (STIs) in GP practices, yet recent increases in the rates of STIs demonstrate that there is more to be done to develop services to meet local need.

\section{The national strategy for sexual health and $\mathrm{HIV}^{2}$}

In her forward to the strategy consultation document, Yvette Cooper, minister for Public Health, acknowledges the successes of the NHS in providing contraceptive and STI treatment and preventive health care. 'Despite these advances, there can be no room for complacency, as there are serious challenges to be met. Evidence suggests that many people lack the information they want and need to make informed choices that would affect their sexual health. There is a clear relationship between sexual ill health, poverty and social exclusion. The quality of service provision remains varied across the country. For all these reasons it is time to re-examine traditional approaches to the way problems associated with sexual health are addressed.' 2

The strategy consultation document proposes a comprehensive definition of sexual health:

'Sexual health is an important part of physical and mental health. It is a key part of our identity as human beings, together with the fundamental human rights to privacy, a family life and living free from discrimination. Essential elements of good sexual health are equitable relationships and sexual fulfilment with access to information and services to avoid the risk of unintended pregnancy, illness or disease. ${ }^{2}$ [1.2]

The Strategy is wide-ranging in its remit and recommendations to improve sexual health in England. I will not discuss in any depth here the sections of the strategy consultation document on health promotion, or 
those specifically dealing with issues around HIV/AIDS treatment and care. I will concentrate on the proposed model for local NHS services that will shape the future for provision of contraceptive and abortion services.

\section{NHS clinical care and the sexual health and HIV strategy}

Within the Strategy the main elements of NHS sexual health services are considered to be contraceptive care and abortion, and the diagnosis, treatment and prevention of STIs. The major clinical providers of these services (general practice, GUM and community family planning and hospital gynaecology) span all sectors of the NHS, with many NHS-funded abortions from not-for-profit non-NHS providers. However, there are many other NHS clinicians who play a part in identifying those who could benefit from sexual health care and supporting their access to services, such as community nurses (e.g.: school nurses, health visitors) and those providing outreach to specific groups within communities.

Each of the services in this multiplicity of providers have their own service ethos and professional cultures presenting challenges in harnessing together all our efforts to improve sexual health care for local communities.

Any strategic view must recognise these factors and the variations of local sociodemography, the size and configuration of the local sexual health provision and the contributions of primary care. Such complexity of components, combined with the current lack of national or local overview of the quality or coverage of the current services, denies any single, simple organisational solution.

A robust model for the future of services must be developmental, building on the skills and effectiveness of the current providers; it must be flexible for adaptation to local needs and resources, and it must encourage the development and implementation of clinical service standards across all providers.

The Modernisation Plan commits the NHS to shaping services around the needs of patients, and the service model proposed in the strategy consultation document starts with the elements of clinical sexual health care that might be needed by individuals of different gender, age and sexual orientation at different stages of their lives. Taking this approach and mapping the skills, facilities and support needed to provide each of these elements has led to the definition of three levels of clinical sexual health care.

The three levels are recognisable within current services, and between them cover all the necessary elements of clinical care. The model is designed to be used to assess local provision, enable discussion of the pattern of provision in relation to local need, establish clear descriptions of the tasks, roles, skills and inter-relationships of the sexual health care providers, and plan future service development.

Commissioners and providers in primary care, acute and community Trusts need to work together to set up a network that provides all three levels of services and meets the needs of their local population. ${ }^{2}$ [4.6] This approach should enable the development of managed service networks, allowing providers to collaborate and plan services jointly and so provide a more comprehensive and effective service. $^{2}[4.5]$

The importance of the availability of open-access, selfreferring sexual health provision to encourage the use of services by all who need them is recognised, and underpins the model described by the strategy consultation document.
Proposed service levels

Level 1 services

\section{Level 1 services}

Examples of elements of clinical care

- Sexual history and support for risk self-assessment

- Information about full range of contraceptive methods and where available

- Provision of oral and injectable hormonal contraception

- Testing symptomatic women for sexually transmitted infections

- Assessment and referral of men with symptoms of sexually transmitted infections

- HIV testing and counselling (with referral pathways)

- Cervical cytology screening and referral

- Pregnancy testing and referral

- Hepatitis B screening and immunisation

These elements of service are within the scope of primary care teams, and are also provided by family planning and/or GUM services. 'The availability and scope of the services that each primary care team offers must be well advertised. Where a primary care practice can't provide Level 1 services, or has a moral objection to doing so, they must make that clear in practice information and make explicit alternative arrangements for their patients through other practices or community services'. ${ }^{2}$ [4.9]

Local commissioners will need to make arrangements with the providers of more specialist services to allow open access to these basic services for those not registered with a GP and those who choose not to consult their GP for sexual health services. To ensure consistent standards and appropriately integrated care, clinicians should develop and implement locally agreed protocols based on guidelines to be established nationally. ${ }^{2}[4.10]$

\section{Level 2 services}

\section{Level 2 services}

\section{Examples of elements of clinical care}

- STI testing for men

- Partner notification

- Long-acting contraception, IUDs, implants

- Management psychosexual problems

- Vasectomy surgery

'Not all the elements of Level 2 sexual health services can be provided easily or economically by every primary care team. Yet local populations in each PCT area will need access to these elements of care. To make services readily available everywhere, PCTs and PCGs need to identify and support primary care teams with a special interest in sexual health that can provide these services to a high standard, or make sure that they are available from other sources - for example local family planning and GUM clinics working in conjunction with general practices.' ${ }^{2}$ [4.11]

Each team providing Level 2 services 'needs the ability to assess and support people with complex sexual health care needs. Teams also need to act as an expert resource for local colleagues working at Level 1. Advertising and promoting these services will help people to know where they can get them, either directly or on the advice of their general practice.' 2 [4.12] 
'Services should be developed to meet local needs and should be tailored to fill gaps in existing service provision. So PCTs and PCGs will need to develop provision of the full range of Level 2 services in collaboration with local existing open access community family planning and GUM services. With the further development of PCTs, and services increasingly focusing on co-operation to meet local needs, there should be further integration of service organisation and delivery. ${ }^{2}[4.14]$

\section{Level 3 services}

\section{Level 3 services}

At this level specialist clinician teams will be taking responsibility for sexual health services needs assessment and supporting provider quality and clinical governance requirements at all levels, as well as providing specialist services.

\section{Examples of elements of clinical care}

- Outreach for STI prevention/contraception

- Specialised STI management/partner notification

- Specialised HIV treatment and care

- Highly specialised contraception

'Specialist clinical teams will deliver the more specialist aspects of care that need to be provided across more than one PCG or PCT. They will have the focus and expertise necessary to serve people who have more complex, chronic or intensive needs.' ${ }^{2}$ [4.15]

'Level 3 services include those for individual patients and those aimed at improving public health, and will be open access wherever possible. For individual patients they will provide specialist genito-urinary medicine, highly specialised contraception for people with complex medical conditions, specialised HIV services, local co-ordination and back up for sexual assault, termination of pregnancy services, and services for people with psychological and sexual problems. ${ }^{2}$ [4.16]

Those responsible for services at Level 3 should make sure that local guidelines are in place and that there is a framework for monitoring and improving practice. Clinicians in these specialist teams will have had higher training in one or more of the fields of sexual health and will act as an expert clinical resource for clinicians working at Levels 1 or 2 . Nurses will have an expanding role at Level 3 as specialists and consultants. ${ }^{2}$ [4.18]

\section{Local clinical service networks}

'All three levels will form part of a local service network, and each will have explicit responsibilities for different parts of the network. At the specialist level, for example, clinical teams will be responsible for supporting the clinical governance requirements at all levels, providing professional training, designing and updating care pathways and developing new services. ${ }^{2}$ [4.19]

'A whole range of professionals plays some part in sexual health services, but often they offer too narrow a service. This can mean that people have to make contact with several different services before they get all the care they need. For example, family planning clinics may provide an excellent contraception service but rarely offer comprehensive advice on avoiding, diagnosing or treating STIs. ${ }^{2}$ [4.20]

This sort of network approach '... requires shared standards of care between providers, and these standards should be defined, implemented and monitored locally and incorporated in clinical governance processes. Providers and commissioners of services should work together with local people to tailor existing guidance on good clinical practice for local use and to make sure it is implemented.' ${ }^{2}$ [4.79]

'Providers and commissioners need to consider standards across the local network for both clinical services and information. Clinical service standards could cover: open access; referrals between services (care pathways); the availability of a full range of clinically effective services; staff training, support, continuing professional development and lifelong learning; and service monitoring, audit and evaluation.' $^{2}$ [4.81]

\section{Information for service users and potential users}

Providing better information to enable access to services is a key theme of the strategy consultation document. This theme runs through the definition of the three levels and local standards for information are suggested: 'information about the choice of services, including which elements of clinical care are available, where and when services are provided, and pathways between them, the access criteria for services, ... information about other sexual health services, reflecting individual risk assessments.' 2 [ 4.82]

'Commissioners and service providers should also ensure that information about local pregnancy counselling and termination services is readily available and widely publicised. Telephone helplines already function as effective access systems in some parts of the country. ${ }^{2}$ [4.34]

'Services will have to meet a number of new standards for access, including the effective advertising and signposting of services. ... many sexual health services are not well advertised and health professionals themselves are not always up to date with the location, timing and range of available sexual health services. People often have to spend too much time and effort searching for information they need quickly.' ${ }^{2}$ [4.39]

As well as information about the availability of services and which type of care each can provide, there is the need for consistent and evidence-based information 'accompanying treatment or care that helps patients to adhere to it.' ${ }^{2}$ [4.82]

\section{Targeted groups}

'Some groups need targeted sexual health information and HIV/STI prevention because they are at higher risk, are particularly vulnerable or have particular access requirements. Strategies need to be developed to respond to the specific information and prevention needs of local populations. They should assess the needs of:

- young people, and especially those in, or leaving care

- black and minority ethnic groups

- gay and bisexual men

- injecting drug misusers

- adults and children living with HIV and other people affected by HIV

- sex workers

- people in prisons and youth offending establishments.' ${ }^{2}$ [3.22]

'In targeting these groups commissioners and providers need to work together to overcome the common barriers to accessing information and prevention services. These include stigma, discrimination, poverty and social exclusion, language, access problems, low awareness and concerns about confidentiality. ${ }^{2}$ [3.23] 


\section{Commissioning the new model for clinical services}

The model proposed for clinical services requires an overview of all local providers of the various components of sexual health services, and the recognition by all providers of the necessity of working more effectively together to serve local needs.

'The job of commissioning the various components of sexual health and HIV services is carried out currently at different levels and by different organisations. From April 2002 PCTs will be able to commission sexual health services. ${ }^{2}$ [5.2] ... every commissioning organisation must be able to show that they have applied these principles of effective commissioning of sexual health and HIV services:

- using a multi-agency and multi-disciplinary steering group to develop and implement a local action plan

- understanding local needs and identifying priority population groups

- linking to the wider policy context

- working in partnership with other agencies and with users

- being centred transparently on community and patient

- identifying current resources, including those that need development

- setting clear local targets for monitoring the development, implementation and outcomes of plans.' 2 [5.3]

'Local stakeholders, including all the organisations responsible for commissioning services, should agree a sexual health and HIV plan. A local multi-agency group should be set up to inform, implement and monitor this planning.' ${ }^{2}$ [5.4]

'Commissioners should identify the current level of investment in sexual health services and HIV, the cost effectiveness of those services and any gaps in resources. Local planning will need to match capacity to need, priorities and targets and set out the resources necessary for meeting the targets. ${ }^{2}$ [5.11]

'Effective partnerships should be at the root of all commissioning of sexual health services. Commissioning plans should incorporate agreed aims and priorities, based on agreed local values and principles, which should reflect those set out nationally. There should be evidence of an effective process for involving stakeholders. ${ }^{2}$ [5.8]

'Commissioners should reflect the aims of this strategy in their local plans. They should:

- reflect the three levels of services, concentrating on the interface between service levels to ensure smooth referrals

- make sure that there are clear open access arrangements, including for people who seek care from GUM services outside their own area

- identify action to meet the needs of targeted groups

- include prevention of HIV and STIs in all service agreements and investment plans
- give local people access to information on services in a variety of ways

- lead the development of managed networks for HIV services and for sexual health services

- define and monitor service standards for providers. ${ }^{2}$ [5.14]

\section{Enabling the new service model}

In order for the new model to be successfully implemented locally, the strategy consultation document recognises five key areas for further development:

- information and data collection

- evidence and research

- professional education and training

- workforce

- finance. $^{2}[6.1]$

'Each of these areas is essential to the strategy and each needs a programme of reform. ${ }^{2}$ [6.2]

All those involved in planning and providing community contraceptive services will no doubt have struggled with these issues locally. Focussed work at central level, which is sensitive to the large variations in local conditions, could be of immense help, as would some more clarity about any extra funding available to support strategy implementation both nationally and locally.

\section{Consultation for strategy development and implementation}

This introduction to the strategy consultation document cannot reflect the breadth or detail of the proposals. I have chosen to concentrate on the themes that will most directly effect provision of contraceptive care. This parochial view will increasingly become less meaningful as services adapt and develop to provide a broader and more appropriate range of clinical care in line with the needs of their local populations.

I would urge you to read the full strategy consultation document with its recommendations (http://www.doh.gov. $\mathrm{uk} / \mathrm{nshs} /$ strategy.htm) and then think about the questions at the end of chapters 3-6. As a member of the strategy steering group I did my best to represent the perspective from community contraceptive services, but now it is your chance to make your views known. Please respond to the consultation document with answers to the questions posed, or with any other comments or suggestions. The address for responses to the consultation is Sex-Health-\&-HIVStrategy@doh.gsi.gov.uk. Comments should arrive no later than 21 December 2001.

\footnotetext{
References

NHS Contraceptive Services, England:1999-2000. Bulletin 2000/27. London: Government Statistical Service, October 2000. http://www doh gov.uk/pdfs/sb0027.pd

The national health strategy for sexual health and HIV. London: Department of Health, July 2001. http://www.doh.gov.uk/nshs/strategy.htm
} 